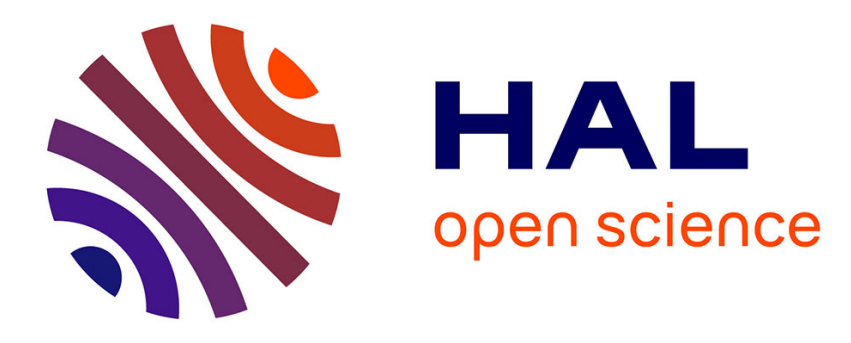

\title{
Dynamical entanglement as a signature of chaos in the semiclassical limit
}

Maurice Lombardi, A. Matzkin

\section{To cite this version:}

Maurice Lombardi, A. Matzkin. Dynamical entanglement as a signature of chaos in the semiclassical limit. Laser Physics, 2010, 20 (5), pp.1215. 10.1134/S1054660X10090343 . hal-00961210

\section{HAL Id: hal-00961210 https://hal.science/hal-00961210}

Submitted on 19 Mar 2014

HAL is a multi-disciplinary open access archive for the deposit and dissemination of scientific research documents, whether they are published or not. The documents may come from teaching and research institutions in France or abroad, or from public or private research centers.
L'archive ouverte pluridisciplinaire HAL, est destinée au dépôt et à la diffusion de documents scientifiques de niveau recherche, publiés ou non, émanant des établissements d'enseignement et de recherche français ou étrangers, des laboratoires publics ou privés. 


\title{
Dynamical entanglement as a signature of chaos in the semiclassical limit
}

\author{
M. Lombardi and A. Matzkin \\ Laboratoire de Spectrométrie physique (CNRS Unité 5588), \\ Université Joseph-Fourier Grenoble-1, \\ BP 87, 38402 Saint-Martin d'Hères, France
}

\begin{abstract}
The relationship between classically chaotic dynamics and the entanglement properties of the corresponding quantum system is examined in the semiclassical limit. Numerical results are computed for a modified kicked top, keeping the classical dynamics constant while investigating the entanglement for several versions of the corresponding quantum system characterized by a different value of the effective Planck constant $\hbar_{\mathrm{eff}}$. Our findings indicate that as as $\hbar_{\mathrm{eff}} \rightarrow 0$, the apparent signatures of classical chaos in the entanglement properties, such as characteristic oscillations in the time-dependence of the linear entropy, can also be obtained in the regular regime. These results suggest that entanglement is not a universal marker of chaotic dynamics of the corresponding classical system.
\end{abstract}

PACS numbers: 03.65.Ud,05.45.Mt,34.60.+z,03.67.Mn 


\section{INTRODUCTION}

There has been a certain degree of ongoing controversy lately concerning the connection between classical chaos and entanglement. Indeed, the quantum-classical correspondence, based on the asymptotics of the Schrödinger equation [1] or on the equivalent approximation in the path integral formulation of quantum mechanics [2], has allowed a successful interpretation of a host of properties of a quantum system in terms of the properties of the corresponding classical system. For example fluctuations in the spectrum of a quantum system are well-known to be related to classical periodic orbits [3]; certain quantum level statistics are in direct correspondence with the average properties of generic regular or chaotic motion [4].

A few years ago, several groups started investigating the links between classically chaotic dynamics and the degree of entanglement of the corresponding quantum system. Initial work [5-7] appeared to indicate that chaos generated a higher and faster degree of entanglement. This claim was subsequently revised, as it was found that some systems, or even some initial states in the previously studied systems, could display as much entanglement when the corresponding classical regime was regular [8-12]. Still, some works [13, 14], although recognizing that chaos is not necessarily associated with higher and faster entanglement, saw a specific signature of classically chaotic motion in the dynamics of entanglement, for example marked oscillations in the time-dependence of the reduced linear entropy. To be fair it must be noticed that there is a certain degree of confusion when comparing works investigating the connection between classical dynamics and entanglement: besides the usual problem of properly identifying the pertinent classical counterpart of a quantum system, most of the works in this area have dealt with systems composed of two coupled sub-systems, each of the two sub-systems being characterized by a regular or chaotic classical regime. However, as it has been previously pointed out $[11,14]$, the coupling between the two sub-systems is the process giving rise to entanglement. Therefore, in our opinion, the most relevant systems to employ in order to understand the connection between chaos and entanglement are those in which the same physical process creates chaos (in the classical system) and entanglement (in its quantum counterpart).

The main goal of this paper is to investigate the signatures of chaos in the entanglement dynamics for such a system as the semiclassical limit is approached. Our system is a modified 
kicked top in which the total angular momentum $\mathbf{J}$ is fixed and results from the coupling of two angular momenta $\mathbf{L}$ and $\mathbf{N}$. From a quantum-mechanical perspective, this coupling gives rise to entanglement. The quantity $1 / L$ plays the rôle of an effective Planck constant $\hbar_{\text {eff }}$. An invariance property of the classical dynamics allows to change $J, L$ and $N$ without affecting the stroboscopic map; in turn this invariance can be employed to investigate the properties of the quantum system as $J, L$ and $N$ are increased, i.e. as $\hbar_{\text {eff }} \rightarrow 0$ while keeping the classical dynamics constant. We will see that the apparent signatures of classically chaotic dynamics visible in the reduced linear entropy in the deep quantum regime tend to vanish as $\hbar_{\mathrm{eff}} \rightarrow 0$. The model employed here, introduced previously [11], will briefly be recalled in Sec. 2. We will compute the time-dependent generation of entanglement for varying $\hbar_{\mathrm{eff}}$ in Sec. 3, and examine more closely the Hamiltonian eigenstates as $\hbar_{\mathrm{eff}} \rightarrow 0$ in Sec. 4. The results will be discussed and summarized in Sec. 5.

\section{THE MODIFIED KICKED TOP: A RYDBERG MOLECULE}

Let $\mathbf{J}$ be the total angular momentum of the system. $J$ and its projection $M$ on an arbitrary axis are fixed and conserved; $\mathbf{J}$ results from the addition of the two coupled angular momenta,

$$
\mathbf{J}=\mathbf{N}+\mathbf{L} .
$$

The coupling can be seen as arising from a kick a light particle (an electron) with angular momentum $\mathbf{L}$ receives from the other much more massive sub-system (the diatomic nuclei) freely rotating with angular momentum $\mathbf{N}$. After the kick the light particle is ejected on an attractive orbit along which it is brought back towards the nuclei for an additional, periodic kick. The Hamiltonian is given by

$$
H=b \mathbf{N}^{2}+\frac{\mathbf{L}^{2}}{2 m r^{2}}+V(k)+H_{r}
$$

where $b$ is a rotational constant and $H_{r}$ is the radial Hamiltonian (containing the attractive potential) of the light particle of mass $m . V(k)$ is the kicking potential whose range is restricted to $r \approx 0$. By making the following additional assumptions, both justifiable on physical grounds (i) $L$ is conserved by the kick; (ii) $L \cos \theta$ is also conserved where $\theta$ is the angle $\mathbf{L}$ makes with the axis going through the two nuclei, $V(k)$ is chosen so that $\mathbf{L}$ can only 


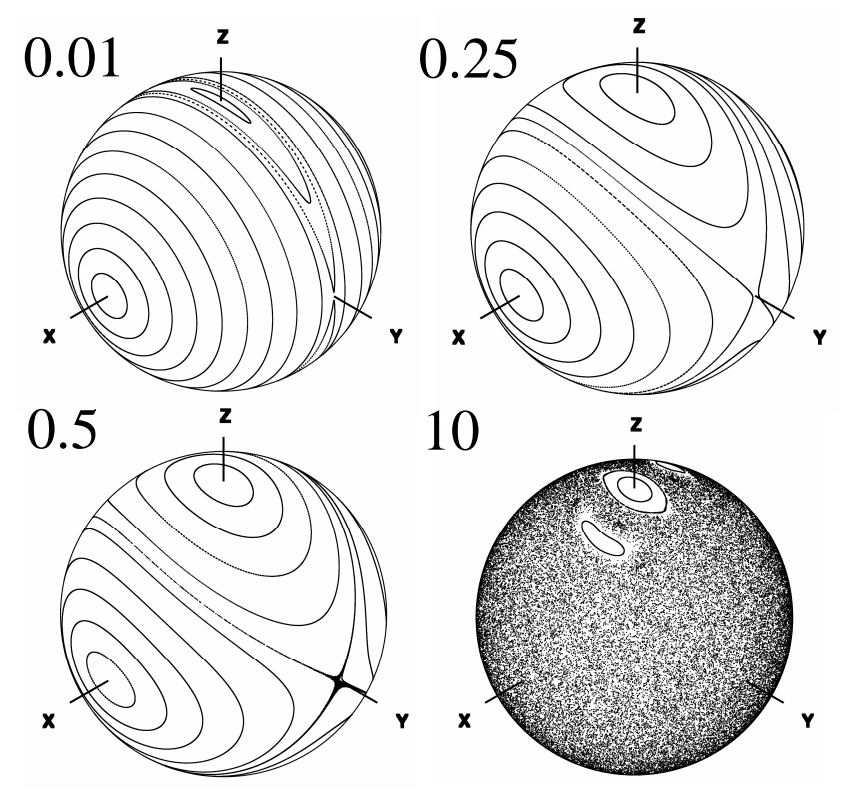

FIG. 1: Poincaré surface of section for different values of the coupling $k=0.01, k=0.25, k=0.5$ and $k=10$. The map shows the position of $\mathbf{L}$ after each kick; $\mathbf{N}$ lies on the $x$ axis, so that a fixed value of $N$ corresponds by Eq. (1) to a constant projection of $\mathbf{L}$ on the $x$ axis. $\theta$ is the angle $\mathbf{L}$ makes with the $z$ axis

rotate by an angle assumed to be given by [15]

$$
\delta \phi=k \cos \theta
$$

Hence $k$ represents the strength of the kick. Since $J$ and $L$ are conserved Eq. (1) implies that $N$ isn't, since the standard addition of angular momenta yield $|J-L| \leq N \leq|J+L|$. A stroboscopic map is obtained by plotting the position of $\mathbf{L}$ after each kick (Fig. 1).

In the quantum mechanical version of the system the kicks defined by $V(k)$ become scattering phase-shifts $\eta_{\lambda}$ depending on $k, \lambda$ denoting the partial wave in the frame rotating with the nuclei. Indeed the rotation $\delta \phi$ defined by Eq. (3) is the deflection angle of the orbit, whose relation to the scattering phase-shifts in the semiclassical limit is well-known to be given by the general relation

$$
\delta \phi=2 \frac{\partial \eta_{\lambda}}{\partial \lambda}
$$

for a full description of the quantum and classical versions of the model employed here and their relations see Ref. [11]. The total energy of the system is

$$
E=b N(N+1)+\epsilon_{N}
$$


where $\epsilon_{N}$ is the energy of the light particle (the excited but bound Rydberg electron) traditionally labeled by the effective quantum number $\nu_{N}$,

$$
\epsilon_{N}=\frac{-1}{2 \nu_{N}^{2}}
$$

(atomic units will now be used throughout). A product state at fixed energy, denoted

$$
\left|\phi_{N}(E)\right\rangle=|N\rangle\left|F\left(\epsilon_{N}\right)\right\rangle
$$

will not be an eigenstate of the Hamiltonian (2) given that $V(k)$ entangles the states $|N\rangle$ and $\left|F\left(\epsilon_{N}\right)\right\rangle$ of the nuclei and electron respectively. Instead, an eigenstate involves the superpositions

$$
|\psi(E)\rangle=\sum_{N} B_{N}(E)\left|\phi_{N}(E)\right\rangle
$$

which only exist for an infinite set of discrete energies $E$ that are found by employing the techniques of quantum defect theory [11].

Note the Poincaré surface of section of the map depends for a fixed value of $k$ only on the relative periods of the light particle orbit and the rotation of the nuclei, which in turn depend of the moment of inertia $I$ of the nuclei and the energy $E$. Hence if the angular momenta $J, L$ and $N$ are increased, the stroboscopic map at fixed $k$ can be kept invariant by adjusting them. The surfaces of section shown in Fig. 1 will stick to this convention: a given surface of section will be characterized solely by the coupling strength $k$, the moment of inertia and the energy of the system being adjusted, depending on the values of $J, L$ and $N$ to their unique values so that the system obeys the map. For the quantum top, this allows to study the correspondence with the classical map as $\hbar_{\text {eff }}$ (the inverse of $L$ ) varies, the energy range in which the quantum levels are computed corresponding to the classical energy keeping the stroboscopic map constant. Obviously as the angular momenta increase, the number of available quantum states also increases. However these additional states are quantized on the same underlying classical dynamical substrate (by adjusting the energy), so that on average one expects the same behavior for the observables obeying the quantum-classical correspondence as $\hbar_{\mathrm{eff}}$ decreases.

\section{ENTANGLEMENT GENERATION}

Entanglement is a distinct quantum feature and has therefore no straightforward marker that would obey the quantum classical correspondence. To quantify entanglement we will 
determine the linear entropy $S_{2}$ associated with the reduced density matrix $\rho_{e}$ descibing the outer electron,

$$
\rho_{e}(t)=\operatorname{Tr}_{N} \rho(t)=\sum_{N}\langle N|\rho(t)| N\rangle,
$$

where $\rho(t) \equiv|\psi(t)\rangle\langle\psi(t)|$ is the density matrix of the system and $\operatorname{Tr}_{N}$ (resp. $\operatorname{Tr}_{e}$ ) refers to averaging over the nuclei (resp. outer electron) degrees of freedom. The reduced linear entropy employed in this work will be defined by

$$
S_{2}(t)=\frac{n}{n-1}\left(1-\operatorname{Tr}_{e} \rho_{e}^{2}(t)\right)
$$

where $n$ is the dimension of the density matrix. The normalization prefactor ensures that $S_{2}$ vanishes for a pure state and is unity for a uniformly mixed state, irrespective of the number of dimensions. We will investigate the time evolution $S_{2}(t)$ starting from an initial (at $t=0)$ product state for 4 regimes of $\hbar_{\mathrm{eff}}: \hbar_{\mathrm{eff}} \approx 1(J=10, L=2$, deep quantum regime), $1 / 5(J=20, L=4$, moderately excited states $), 1 / 10(J=50, L=10$, excited states) and $1 / 100(J=500, L=100$, semiclassical regime).

The computations proceed as in [11]: we assume that at $t=0$ the system is in a well defined rotational state $\left|N_{0}\right\rangle$ whereas the outer electron is radially localized at the outer turning point of the Kepler orbit (of period $T_{e}$ ), several thousand atomic units away from the nuclei. Hence

$$
\psi(t=0, r)=F_{l o c}\left(r \approx r_{t p}\right) \otimes\left|N_{0}\right\rangle
$$

where $F_{l o c}(r)$ has a Gaussian envelop and matches the energy of the corresponding classical regime. The wavepacket attracted by the Coulomb interaction moves towards the nuclei and collides at $t \approx T_{e} / 2$. At later times the wavefunction is given by

$$
|\psi(t)\rangle=\sum_{E} \sum_{N} \mathcal{B}_{N}(E) e^{-i E t}\left|F\left(\epsilon_{N}\right)\right\rangle|N\rangle
$$

where

$$
\mathcal{B}_{N}(E)=B_{N}(E) B_{N_{0}}(E)\left\langle F\left(\epsilon_{N}\right) \mid F_{l o c}\right\rangle
$$

Note that the scattering nature of the formalism introduces a slight non-Hermiticity that cannot be neglected and thus needs to be explicitly taken into account [16]. This is one of the reasons that makes the numerical computations very involved as $\hbar_{\text {eff }}$ decreases and the basis sizes depending on $n$ increase. 

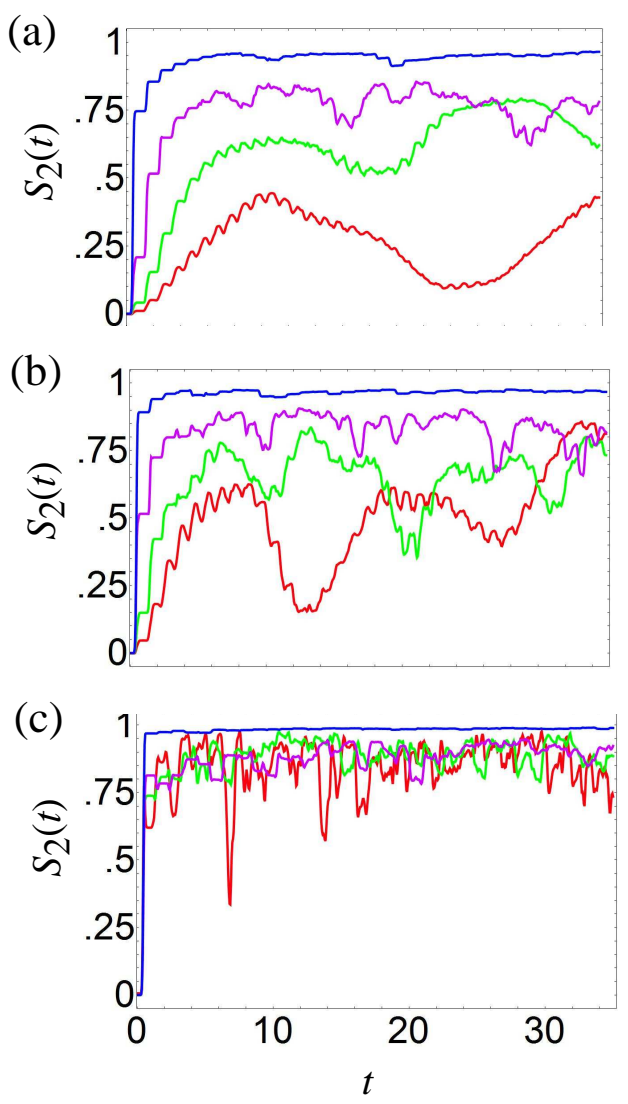

FIG. 2: The linear entropy as a function of time (with units parameterized by the number of kicks) for varying values of $\hbar_{\mathrm{eff}}: \hbar_{\mathrm{eff}} \approx 1$ (red curve), $\hbar_{\mathrm{eff}} \approx 1 / 5$ (green), $\hbar_{\mathrm{eff}} \approx 1 / 10$ (purple) and $\hbar_{\mathrm{eff}} \approx 1 / 100$ (blue line), and for different values of $k$. (a): $k=0.25 ;(\mathrm{b}): k=0.5 ;(\mathrm{c}): k=10$.

Fig. 2 shows the time evolution of the entanglement for the 4 values of $\hbar_{\text {eff }}$ mentioned above. The initial state $\left|N_{0}\right\rangle$ lies on a ring centered on the $x$ axis slightly behind the origin. Fig. 2(a) presents the linear entropy $S_{2}(t)$ for $k=0.25$, each of the 4 curves corresponding to a different value of $\hbar_{\text {eff }}$. The time is given as a function of the period of the central Kepler orbit, i.e. it reflects the number of kicks which is the same in all the cases, whereas the timescale (the period of the orbits), being proportional to an inverse power of $\hbar_{\mathrm{eff}}$, is of course different for each value of $\hbar_{\mathrm{eff}}$.

Figs 2(b) and (c) show $S_{2}(t)$ for $k=0.5$ and $k=10$ respectively (the corresponding classical dynamical regimes are given by the surfaces of section of Fig. 1). The results for the lowest $\hbar_{\mathrm{eff}}$ values were given previously in $[10,11]$ (beware however of the different normalization of the linear entropy employed in those works, since only the situation for a fixed value of $\hbar_{\text {eff }}$ was considered); we discussed there the behavior of the entanglement 


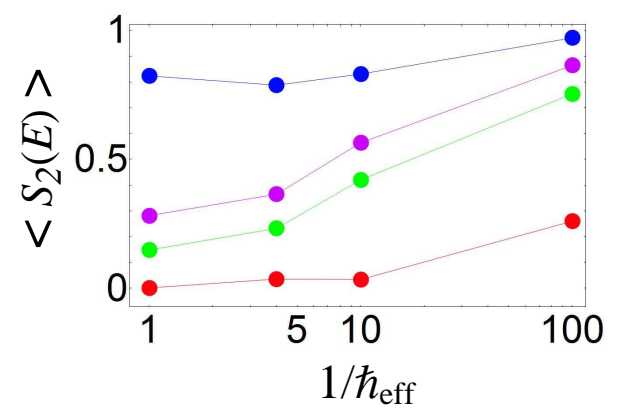

FIG. 3: The average linear entropy is given as a function of $\hbar_{\mathrm{eff}}$ for the 4 values of $k$ whose corresponding classical surfaces of section were shown in Fig. 1. From bottom to top $k=0.01$ (red dots) $k=0.25$ (green), $k=0.5$ (purple) and $k=10$ (blue dots). The lines connecting the dots are displayed only for visual convenience.

generation when $k$ varied, for a single fixed value of $\hbar_{\mathrm{eff}}$. The focus here is on the variation of $S_{2}(t)$ when $\hbar_{\text {eff }} \rightarrow 0$ but the classical regime is fixed. When the corresponding classical regime is regular [Figs. 2(a)-(b)] we see a dramatic increase in the entanglement rate: for the higher values of $\hbar_{\text {eff }}$ the linear entropy increases progressively after each kick, whereas for $\hbar_{\text {eff }} \approx 1 / 100$ it takes only 2 or 3 kicks for $S_{2}(t)$ to reach its maximal, significantly higher, value. This behavior was generally thought to be typical of classically chaotic corresponding systems. Note also that the smooth oscillations, clearly visible in the deep quantum regime $\left(\hbar_{\mathrm{eff}} \approx 1 / 2\right.$ and $\left.1 / 5\right)$, which are generally taken to be characteristic of a classically regular regime, disappear as $\hbar_{\mathrm{eff}} \rightarrow 0$. When the dynamical regime of the corresponding classical system is chaotic (Fig. 2(c), $k=10$ ) the violent oscillations produced by a single kick tend to disappear as $\hbar_{\text {eff }} \rightarrow 0$. For $\hbar_{\text {eff }} \approx 1 / 100 S_{2}(t)$ reaches its saturation maximum after a single kick, and stays approximately constant. In the deep quantum regime, the low dimensionality of the Hilbert space $(n=3)$ allows recombinations in which one of the channels is depleted to occur periodically, giving rise to the dips visible in the $\hbar_{\mathrm{eff}} \approx 1 / 2$ curve.

\section{ENTANGLEMENT OF THE EIGENSTATES}

The eigenstates $|\psi(E)\rangle$ of a Rydberg molecule are given by Eqs. (7)-(8) showing each $|\psi(E)\rangle$ is a superposition of product states. We compare here the degree of entanglement of a bunch of eigenstates for a given value of $k$ corresponding to the same classical dynamics as $\hbar_{\text {eff }}$ varies. To do so, we compute the reduced linear entropy for each eigenstate, defined 
by

$$
S_{2}(E)=\frac{n}{n-1}\left(1-\operatorname{Tr}_{e} \rho_{e}^{2}(E)\right)
$$

where $\rho_{e}(E)$ is obtained similarly as in Eq. (9) by tracing out the nuclear degrees of freedom from the eigenstate $\rho(E) \equiv|\psi(E)\rangle\langle\psi(E)| . \quad S_{2}(E)$ ranges from 0 for a product state to 1 for an eigenstate having all the $B_{N}$ coeficients of Eq. (8) equal. The average $\left\langle S_{2}(E)\right\rangle$ is computed on an energy range over which the classical dynamics is constant; hence in the deep quantum regime, the average involves a limited number of states (less than 20) whereas for $J=500$ several hundred states are quantized within the same energy range.

Fig. 3 shows $\left\langle S_{2}(E)\right\rangle$ for 4 different values of $k$ as $\hbar_{\text {eff }}$ is varied. The increase of the mean linear entropy as $\hbar_{\mathrm{eff}} \rightarrow 0$ is spectacular: even for $k=0.01$ near the integrable limit, the eigenstates become significantly mixed as $J$ and $L$ increase. A better understanding is gained by looking at $S_{2}(E)$ for individual states. This is done in Fig. 4 . The left panel shows the linear entropy for $k=0.01$ when $\hbar_{\text {eff }} \approx 1$ [Fig. 4(a)] and $\hbar_{\text {eff }} \approx 1 / 100$ [Fig. 4(b)], each dot giving the value $S_{2}(E)$ for an eigenstate. The right panel gives the same quantities but for $k=0.5$. Both values of $k$ correspond to classical regular regimes (Fig. 1).

For $k=0.01$ the classical map tends to conserve the value of $N$ (the rings of the Poincaré surface of section are centered on the $\mathbf{N}$ axis). We see nevertheless that as $\hbar_{\mathrm{eff}} \rightarrow 0$ some eigenstates present a high degree of entanglement - actually intertwined series of a doublet of states with higher entanglement than the rest are visible as $E$ varies. This behavior, which is also apparent in the $k=0.025$ case (not shown here) can be explained by considering that quantization does not necessarily take place on the rings encircling the $N$ axis. For instance a group of eigenstates tends to quantize on rings encircling the fixed point on top of the $z$ axis, hence with a spread in $N$. In the deep quantum regime this spread in $N$ is contained within the area occupied by a single rotational eigenstate $|N\rangle$ on the sphere; but as $J, L$ and $N$ increase the relative width of a ring representing the single rotational state $|N\rangle$ on the unit sphere decreases and an eigenstate quantized around the $z$ axis will span an area overlapping with the area covered by several $|N\rangle$ states, and will therefore present a high degree of entanglement. For $k=0.5$ most of the points on the surface of section tend to induce classical dynamics that change the value of $N$. Hence even in the deep quantum regime individual eigenstates will present a varying degree of entanglement and as $\hbar_{\mathrm{eff}} \rightarrow 0$ these eigenstates will spread over a higher number of rotational states $|N\rangle$. Indeed the linear entropy, which shows important variations among the individual states in Fig. 4(c) tends to 
(a)

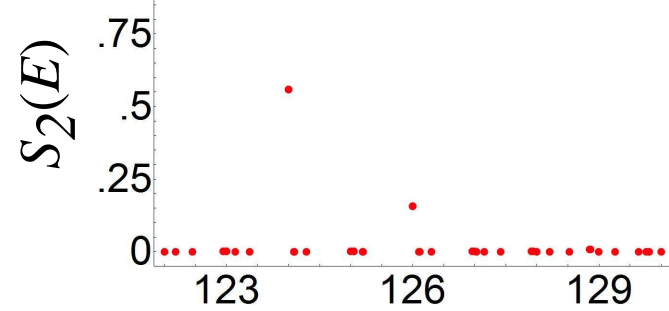

(b)

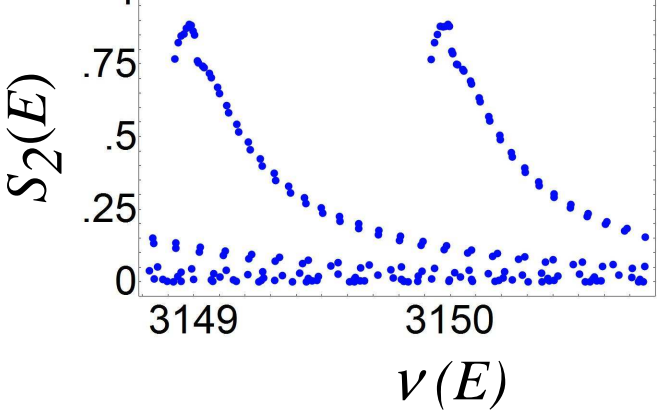

(c)

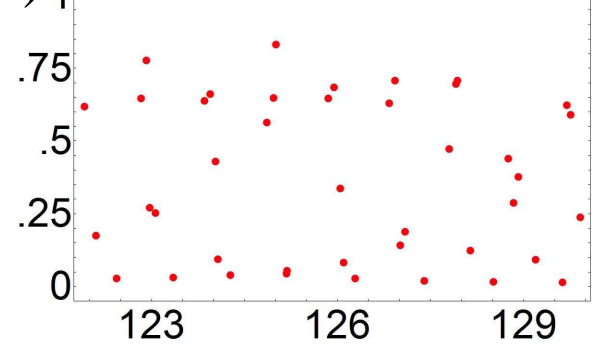

(d)

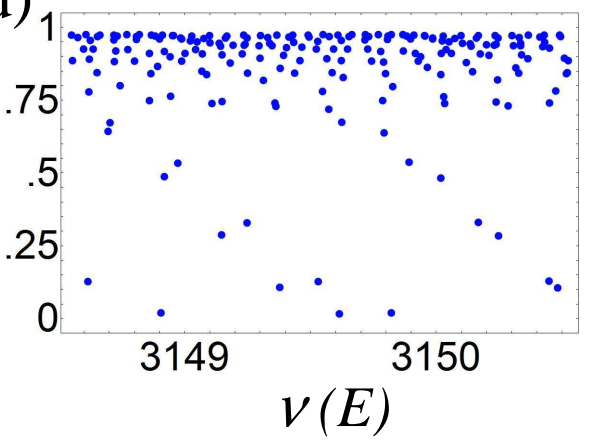

FIG. 4: The linear entropy for the individual eigenstates of the Hamiltonian over an energy range corresponding to constant classical dynamics. Each dot corresponds to an individual eigenstate. The energy is parameterized by $\nu(E)$ [see Eq.(6)]. (a) and (b) give $S_{2}(E)$ for $k=0.01$, for the case $\hbar_{\mathrm{eff}} \approx 1(\mathrm{a})$ and $\hbar_{\mathrm{eff}} \approx 1 / 100(\mathrm{~b}) .(\mathrm{c})$ and $(\mathrm{d})$ give $S_{2}(E)$ for $k=0.5$, for the case $\hbar_{\mathrm{eff}} \approx 1$ (c) and $\hbar_{\mathrm{eff}} \approx 1 / 100(\mathrm{~d})$.

accumulate near its maximal value for $\hbar_{\mathrm{eff}} \approx 1 / 100$ [Fig. 4(d)].

\section{DISCUSSION AND CONCLUSION}

We have determined the entanglement dynamics for varying values of $\hbar_{\text {eff }}$ of a typical Rydberg molecule, i.e. a modified kicked top whose classical corresponding system displays classically regular or chaotic regimes depending on the coupling constant $k$ between the angular momenta. The present findings confirm that entanglement can be as effective when the corresponding classical dynamics is regular than when the regime is classically chaotic. Moreover, even when there is a difference in the entangling power, this difference tends to decrease as $\hbar_{\mathrm{eff}} \rightarrow 0$ (compare in Figs. $2(\mathrm{a})$ and $2(\mathrm{c})$ the blue $\hbar_{\mathrm{eff}} \approx 1 / 100$ curves with the red $\hbar_{\text {eff }} \approx 1$ ones). This is a consequence of the fact that for a given value of $k$, the entanglement rate increases as $\hbar_{\text {eff }}$ decreases. A partial explanation for this behavior was given in Sec. 4: as 
the dimensionality of the Hilbert space increases, the states tend to quantize over a greater number of channels; put differently, an equivalent kick strength will send the incoming wave over a significantly higher number of states $|N\rangle$, creating more entanglement. This effect is expected to be stronger when the corresponding classical dynamics is regular, since the probability distribution moves smoothly on the sphere; as $\hbar_{\mathrm{eff}}$ decreases, this distribution lies in the same region on the sphere but overlaps with a greater number of $|N\rangle$ states (recall that each $|N\rangle$ state occupies a ring around the $x$ axis whose width is proportional to $\hbar_{\text {eff }}$ ). Instead when the map is chaotic, different bits of the distribution tend to appear randomly on the sphere, so that the overlap with the number of $|N\rangle$ states (normalized relative to the total number of available states) will not increase significantly as $\hbar_{\text {eff }}$ varies.

The second important conclusion one can draw from the present results concerns the issue of a specific signature of chaos in the entanglement properties. While it is indisputable that, provided the mechanism that creates chaos in the quantum system is the same that the one that creates entanglement in the quantum one, a chaotic map will generically induce a high entangling power, it is doubtful that a universal signature of chaos can be found in the generation of entanglement. The oscillations in $S_{2}(t)$ that have sometimes $[13,14]$ been associated with a classically regular regime are in fact the visible result of partial revivals in low dimensional Hilbert spaces. As the dimensionality of the Hilbert space increases, which is indeed the case as $\hbar_{\mathrm{eff}} \rightarrow 0$, the revivals will not be visible when multiple interferences between channels having equal weights in the total wavefunction occur. This is precisely the situation for highly entangled states in the regular regime: the oscillations in Figs. 2(a) and (b), showing the entanglement rate for $k=0.25$ and $k=0.5$ respectively, become less important as $\hbar_{\text {eff }}$ decreases, and are hardly visible at all for the blue $\hbar_{\text {eff }} \approx 1 / 100$ curve. By inspecting the blue curve of Fig. 2(b) (classically regular) and that of Fig. 2(c) (classically chaotic dynamics), it is hardly possible to identify the entanglement dynamics with a specific classical regime.

Summarizing, we have investigated the relationship between the entanglement properties of a modified kicked top (modeling a Rydberg molecule) and the dynamics of the corresponding classical system as the semiclassical limit is approached. This was done by working with quantum systems characterized by a lower value of $\hbar_{\text {eff }}$ while keeping the dynamics of the stroboscopic map constant. Our results indicate that the linear entropy increases as $\hbar_{\mathrm{eff}} \rightarrow 0$, in particular in the regular regime. Moreover for highly entangled states, the behavior of 
the linear entropy as the semiclassical limit is approached does not discriminate between classically regular and chaotic corresponding systems. These findings can be qualitatively explained by drawing on arguments grounded on the classical-quantum correspondence.

[1] V. P. Maslov and M. V. Fedoriuk, Semi-Classical Approximation in Quantum Mechanics (Reidel, Dordrecht, 1981).

[2] M. C. Gutzwiller, Chaos in Classical and Quantum Mechanics (Springer, Berlin, 1990) .

[3] M. Brack and R. Bhaduri, Semiclassical Physics (Addison-Wesley, Reading, MA, 1997).

[4] F. Haake Quantum signatures of chaos (Springer, Berlin, 2004).

[5] P. A. Miller, and S. Sarkar Phys. Rev. E 60, 1542 (1999).

[6] A. Lakshminarayan Phys. Rev. E 64, 036207 (2001).

[7] A. Lahiri and S. Nag, Phys. Lett. A 318, 6 (2003).

[8] H. Fujisaki Phys. Rev. A 70, 012313 (2004).

[9] R. Demkowicz-Dobrzanski, and M. Kus Phys. Rev. E 70, 066216 (2004).

[10] M. Lombardi and A. Matzkin, Europhys. Lett. 74, 771 (2006).

[11] M. Lombardi and A. Matzkin, Phys. Rev. A 73, 062335 (2006).

[12] S. Zhang and Q. Jie, Phys. Rev. A 77012312 (2008).

[13] X. Wang, S. Ghose, B. C. Sanders and B. Hu Phys. Rev. E 70, 016217 (2004).

[14] C. M. Trail, V. Madhok and I. H. Deutsch, Phys. Rev. E 78, 046211 (2008).

[15] M. Lombardi, P. Labastie, M. C. Bordas, and M. Broyer, J. Chem. Phys. 89, 3479 (1988).

[16] A. Matzkin, J. Phys. A 39, 10859 (2006). 